\title{
NCAPG confers trastuzumab resistance via activating SRC/STAT3 signaling pathway in HER2-positive breast cancer
}

\author{
Lili Jiang 1,2, Liangliang Ren ${ }^{3}$, Han Chen 1,2, Jinyuan Pan 1,2, Zhuojun Zhang 1,2, Xiangqin Kuang 1,2, Xuhong Chen ${ }^{1,2}$, \\ Wenhao Bao ${ }^{1,2}$, Chun Lin ${ }^{1,2}$, Zhongqiu Zhou ${ }^{1,2}$, Danping Huang ${ }^{4}$, Jianan Yang ${ }^{1,5}$, Hongbiao Huang ${ }^{1,2}$, Lan Wang ${ }^{6}$, \\ Ning $\mathrm{Hou}^{7}$ and Libing Song ${ }^{8}$
}

\begin{abstract}
HER2+ breast cancer (BC) is characterized by rapid growth, early recurrence, early metastasis, and chemoresistance. Trastuzumab is the most effective treatment for HER2 $+B C$ and effectively reduces the risk of recurrence and death of patients. Resistance to trastuzumab results in cancer recurrence and metastasis, leading to poor prognosis of HER2+ BC. In the present study, we found that non-structural maintenance of chromosome condensin 1 complex subunit $G$ (NCAPG) expression was highly upregulated in trastuzumab-resistant HER2+ BC. Ectopic NCAPG was positively correlated with tumor relapse and shorter survival in HER2+ BC patients. Moreover, overexpression of NCAPG promoted, while silencing of NCAPG reduced, the proliferative and anti-apoptotic capacity of HER2+ BC cells both in vitro and in vivo, indicating NCAPG reduces the sensitivity of HER2 + BC cells to trastuzumab and may confer trastuzumab resistance. Furthermore, our results suggest that NCAPG triggers a series of biological cascades by phosphorylating SRC and enhancing nuclear localization and activation of STAT3. To summarize, our study explores a crucial role for NCAPG in trastuzumab resistance and its underlying mechanisms in HER2+ BC, and suggests that NCAPG could be both a potential prognostic marker as well as a therapeutic target to effectively overcome trastuzumab resistance.
\end{abstract}

\section{Introduction}

Breast cancer $(\mathrm{BC})$ remains the leading cause of morbidity and mortality among cancers in females worldwide $^{1}$. Based on gene expression profiling and molecular pathology, BC was traditionally classified into luminal A, luminal $\mathrm{B}$, human epidermal growth factor receptor 2 positive (HER2+), and Basal-like type subtypes ${ }^{2,3}$. HER2+

\footnotetext{
Correspondence: Lili Jiang (jianglili@gzhmu.edu.cn) or

Libing Song (songlb@sysucc.org.cn)

${ }^{1}$ Affiliated Cancer Hospital \& Institute of Guangzhou Medical University, 510095 Guangzhou, China

${ }^{2}$ Guangzhou Municipal and Guangdong Provincial Key Laboratory of Protein Modification and Degradation, School of Basic Medical Science, Guangzhou Medical University, 511436 Guangzhou, China

Full list of author information is available at the end of the article

These authors contributed equally: Lili Jiang, Liangliang Ren, Han Chen, Jinyuan Pan

Edited by S. Tait
}

$\mathrm{BC}$ accounts for $15-20 \%$ of total cases of $\mathrm{BC}$, and is characterized by a high degree of malignancy and strong invasiveness, high resistance to chemotherapy, poor effectiveness of endocrine therapy, early recurrence and metastasis, and poor prognosis ${ }^{4,5}$.

Trastuzumab, a humanized HER2-targeting drug, is the most effective treatment for HER2+ BC. Trastuzumab effectively reduces the risk of recurrence and death, extends the disease-free and non-progressive survival period, delays the recurrence time of metastasis, and prolongs survival particularly in patients with metastasis $^{6,7}$. However, the resistance rate of trastuzumab is $66-88 \%$ as a single agent and $20-50 \%$ when combined with chemotherapy in HER2 $+\mathrm{BC}$, and trastuzumab resistance is the major cause of treatment failure resulting in relapse or death ${ }^{8,9}$. Therefore, it is critical to investigate

\section{(c) The Author(s) 2020}

(c) (i) Open Access This article is licensed under a Creative Commons Attribution 4.0 International License, which permits use, sharing, adaptation, distribution and reproduction c. in any medium or format, as long as you give appropriate credit to the original author(s) and the source, provide a link to the Creative Commons license, and indicate if changes were made. The images or other third party material in this article are included in the article's Creative Commons license, unless indicated otherwise in a credit line to the material. If material is not included in the article's Creative Commons license and your intended use is not permitted by statutory regulation or exceeds the permitted use, you will need to obtain permission directly from the copyright holder. To view a copy of this license, visit http://creativecommons.org/licenses/by/4.0/. 
the molecular mechanisms underlying trastuzumab resistance in HER2+ BC in order to improve therapeutic effectiveness and prognosis of patients.

Non-structural maintenance of chromatin condensin 1 complex subunit G (NCAPG) is a subunit of condensin 1 . Human NCAPG was purified initially from HeLa cell nuclear extracts and plays a vital role in condensin activation by regulation of ATPase activity ${ }^{10,11}$. As a tumorpromoting gene, NCAPG has been found overexpressed in several malignancies, including prostate cancer, pediatric high-grade gliomas, and hepatocellular carcinoma $(\mathrm{HCC})^{12-14}$. Upregulation of NCAPG has been suggested to facilitate cell proliferation in gastric cancer ${ }^{15}$. Moreover, ectopic NCAPG was shown to promote cell proliferation, metastasis, differentiation in HCC and was associated with poor TNM stages, poor survival, and tumor recurrence in HCC patients ${ }^{16,17}$. Although NCAPG has previously been reported to be overexpressed and correlated with poor survival in $\mathrm{BC}^{18,19}$, its biological role and mechanism have not been explored.

In the present study, we found that NCAPG was significantly overexpressed in trastuzumab-resistant HER2+ $\mathrm{BC}$ samples and upregulation of NCAPG correlated with poor survival and relapse in HER2+ BC patients. Overexpression of NCAPG was found to promote cell proliferation and decrease trastuzumab-induced apoptosis, while silencing NCAPG re-sensitized resistant HER2+ BC cells to trastuzumab. Furthermore, the SRC/STAT3 pathway was identified to be activated with NCAPG overexpression and mediated trastuzumab resistancerelated cellular functions. These results reveal a critical role for NCAPG in conferring trastuzumab resistance and suggest that NCAPG may be a potential prognostic factor, and therapeutic target, against trastuzumab resistance in HER2+ BC.

\section{Results}

\section{NCAPG is upregulated in trastuzumab-resistant BC}

The Cancer Genome Atlas (TCGA) data analysis displayed that NCAPG was markedly upregulated in BC tissues, particularly in HER2 + cancer tissues $(P<0.001)$ (Fig. S1a, b). To further investigate clinical significance and biological role of NCAPG in the trastuzumabresistant $\mathrm{BC}$, its expression level in HER2+ $\mathrm{BC}$ biopsies with different tolerance to trastuzumab treatment was examined. The results showed that NCAPG was significantly upregulated in trastuzumab-resistant biopsies compared to trastuzumab-sensitive and normal tissues (Fig. 1a, b). NCAPG expression was also significantly increased in the trastuzumab-resistant $\mathrm{BC}$ cell lines SKBR3/TR and BT474/TR compared to parental SKBR3 and BT474 cell lines (Fig. S2). As trastuzumab resistance results in higher tumor relapse rates of HER2 $+\mathrm{BC}^{20}$, we next assessed the expression of NCAPG in patients with

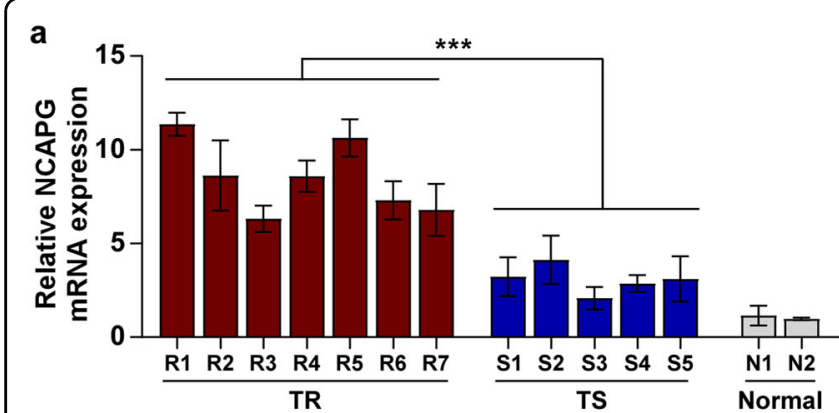

b

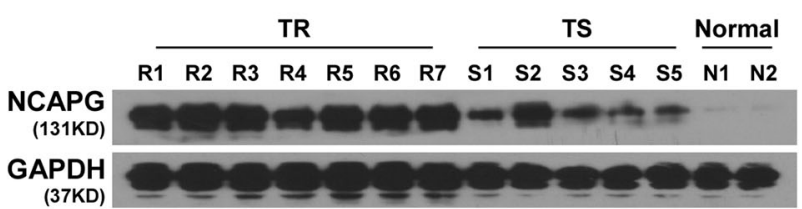

C

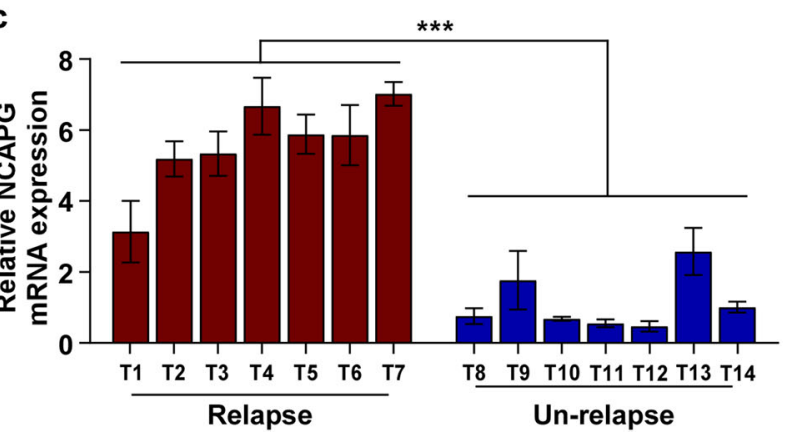

d

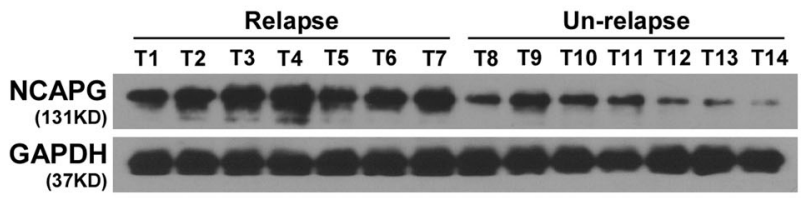

Fig. 1 NCAPG is overexpressed in trastuzumab-resistant breast cancer. $\mathbf{a}, \mathbf{b}$ NCAPG expression was analyzed by qPCR a and western blotting $\mathbf{b}$ in two normal breast tissues and 12 breast cancer specimens, including seven samples of trastuzumab-resistant (TR) and five samples of trastuzumab-sensitive (TS) patients. $\mathbf{c}, \mathbf{d}$ qPCR $\mathbf{c}$ and western blotting results $\mathbf{d}$ of NCAPG expression in BC tissues from patients with or without relapse. Each bar represents the mean \pm SD of three independent experiments. ${ }^{* * *} P<0.001$. 
different relapse status. Both the levels of NCAPG mRNA and protein were remarkably increased in freshly collected $\mathrm{BC}$ tissues from patients with tumor relapse compared to patients without relapse (Fig. 1c, d). These findings suggest that NCAPG upregulation is associated with, and may contribute to, the promotion of trastuzumab resistance in HER2+ BC.

\section{High NCAPG expression correlates with poor prognosis in BC patients}

Immunohistochemistry (IHC) was used to assess NCAPG expression in 103 BC tissue specimen with trastuzumab therapy and six normal paraffin-embedded breast tissues (Supplementary Table S1). NCAPG expression was low or nearly non-detectable in all normal breast tissues (Fig. 2a). In contrast, BC tissues specimen revealed a broader spectrum of expression. Levels of NCAPG were negative $(0)$ in 21 cases, weak $(+1)$ in 23 , moderate $(+2)$ in 27 , and strong $(+3)$ in 32 cases. A correlation analysis showed that NCAPG expression was positively correlated with the age $(P=0.009)$, clinical stage $(P=0.003)$, tumor size (T classification, $P=0.022$ ), metastasis (M classification, $P=0.014)$, vital status $(P<0.001)$, as well as the relapse status $(P<0.05)$ (Fig. $2 \mathrm{~b}$, Supplementary Table S2). In addition, the Kaplan-Meier survival curves and log-rank tests indicated that patients of NCAPG overexpression had a significantly poorer overall survival $(P<0.001)$ and relapse-free survival $(P<0.001)$ (Fig. $2 \mathrm{c})$. Consistently, the analysis resulting from the public database Kaplan-Meier Plotter $^{21}$ (http://kmplot.com/analysis) revealed that BC patients of higher NCAPG expression were correlated significantly with poorer overall, distant metastasis-free and relapse-free survival (Fig. S3). Univariate $(P<0.001)$ and multivariate $(P=0.013)$ analyses showed that NCAPG was an independent prognostic factor in patients with trastuzumab-resistant BC (Supplementary Table S3). To summarize, NCAPG may represent a potential unfavorable prognostic marker in trastuzumab-resistant BC.

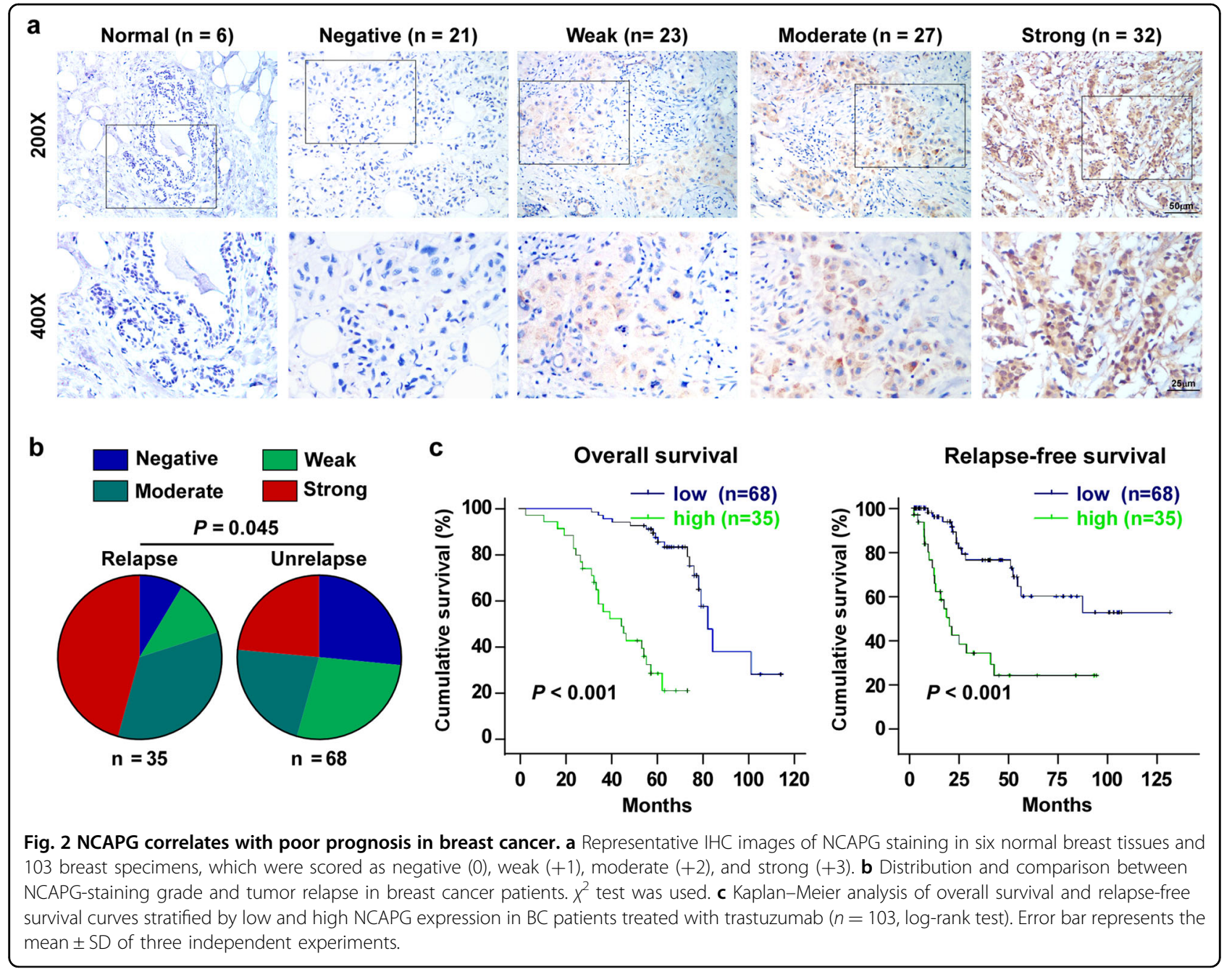



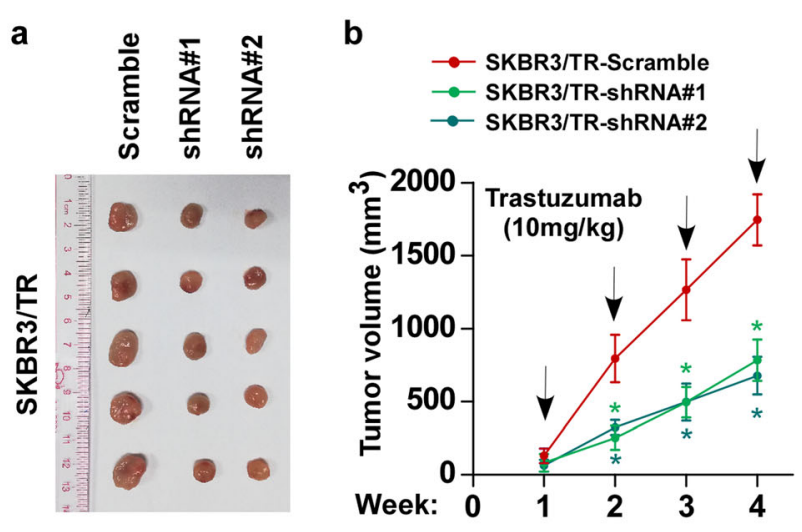

C

d
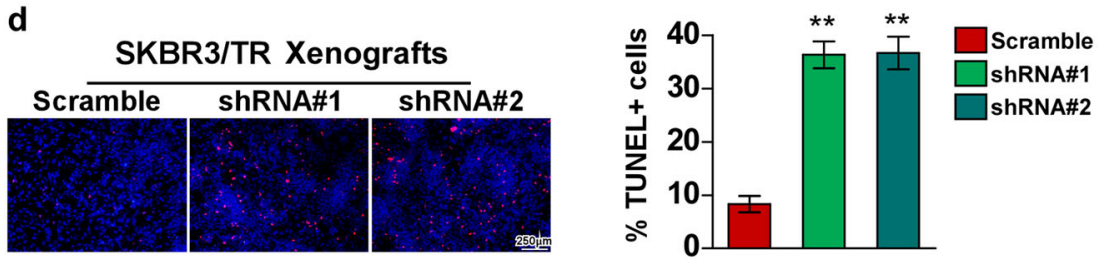

Fig. 3 Knockdown of NCAPG sensitizes resistant HER2 + BC cells to trastuzumab in vivo. a SKBR3/TR-Scramble, SKBR3/TR-shRNA\#1, and SKBR3/ TR-shRNA\#2 were subcutaneously injected into BALB/C nude mice ( $n=5$ mice/group). When the average size of the tumor reached $100 \mathrm{~mm}^{3}$, the mice were treated with an intraperitoneal injection of trastuzumab $(10 \mathrm{mg} / \mathrm{kg}$, once a week) for 4 weeks. b The tumor volumes were examined weekly. c Tumor weights. $\mathbf{d}$ Representative fluorescence images (left) and quantification (right) of TUNEL-stained cells in xenografts formed by the indicated cells treated with trastuzumab. A two-tailed t-test was used for the statistical analysis. Error bar represents the mean \pm SD of three independent experiments. ${ }^{*} P<0.05 ;{ }^{*} P<0.01$.

NCAPG knockdown re-sensitizes trastuzumab-resistant BC cell in vivo

To explore the biological function of NCAPG in trastuzumab-resistant BC cells, NCAPG was silenced in the trastuzumab-resistant $\mathrm{BC}$ cell line SKBR3/TR using two independent short-hairpin constructs (Fig. S4a, b). Xenograft experiment was used to investigate the role of NCAPG in trastuzumab resistance in vivo. SKBR3/TR and NCAPG-silenced SKBR3/TR cells were subcutaneously administered into nude mice. During treatment with trastuzumab, the tumors formed by SKBR3/TR cells retained a higher growth rate, revealing a considerable degree of trastuzumab resistance (Fig. $3 \mathrm{a}-\mathrm{c}$ ). In contrast, the capacity of tumor growth by cells with NCAPG silencing (SKBR3/TR-shRNA\#1/2) was strongly hampered in mice, indicating that NCAPG expression was crucial for trastuzumab resistance in SKBR3/TR cells. Simultaneously, the pro-apoptotic activity of trastuzumab was enhanced by silencing NCAPG (Fig. 3d). Thus, these results indicate that silencing NCAPG re-sensitizes resistant HER2 + BC cells towards trastuzumab therapy.

NCAPG confers trastuzumab resistance in HER2 + BC cells in vitro

The biological role of NCAPG in BC resistance to trastuzumab was also investigated in vitro. Colony formation and cell viability analyses revealed that knockdown NCAPG re-sensitizes SKBR3/TR and BT474/ TR cells to trastuzumab treatment (Fig. 4a, b). As observed in vivo, the pro-apoptotic activity of trastuzumab treatment was increased in SKBR3/TR and BT474/ TR cells with silenced NCAPG expression (Fig. 4c, d). Furthermore, the cell lines stably overexpressing NCAPG, SKBR3-NCAPG, and BT474-NCAPG, were established for further study (Fig. S5). Consistent with the previous results, the proliferative ability of SKBR3-NCAPG and BT474-NCAPG was significantly enhanced, even after trastuzumab treatment, compared to control cells (Fig. 5a, b). Moreover, Annexin V/FITC and TUNEL staining assays both showed that trastuzumab-induced apoptosis of SKBR3 and BT474 cells was significantly reduced by NCAPG overexpression (Fig. 5c, d). These findings indicate that ectopic NCAPG expression may confer trastuzumab resistance in $\mathrm{BC}$ cells.

\section{NCAPG activates SRC/STAT3-signaling pathway}

To investigate the molecular mechanism underlying the ability of NCAPG to confer trastuzumab resistance in BC, gene set enrichment analysis (GSEA) in the published BC dataset was performed. As shown, the expression level of NCAPG was positively correlated with SRC-related gene signatures, suggesting NCAPG might be involved in activation of SRC signaling (Fig. 6a). In line with this hypothesis, western blotting showed that NCAPG 


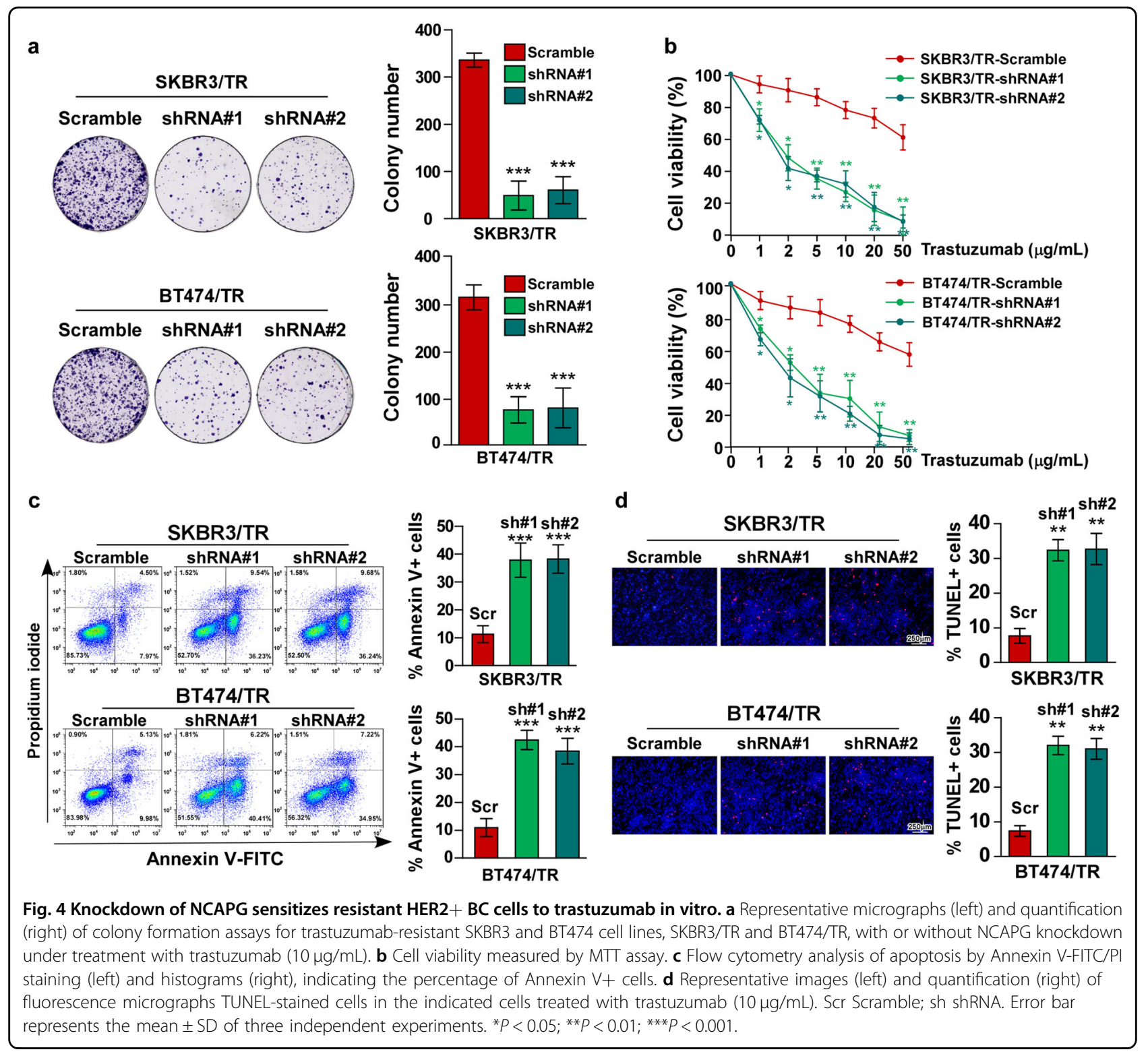

overexpression increased, while silencing NCAPG decreased, phosphorylation of SRC (Tyr416), indicating NCAPG promotes SRC signaling activation (Fig. 6b). Accordingly, the promotion of the proliferative capacity of cells by NCAPG was weakened by inhibiting SRC using a specific inhibitor or shRNA (Fig. 6c), indicating that SRC activity is essential for NCAPG-dependent trastuzumab resistance. The transcription factor STAT3 is downstream of the SRC pathway, and has previously been shown to be correlated with drug resistance of tumors ${ }^{22}$. Therefore, the activity of STAT3 was also examined. The transcriptional activity of STAT3 was enhanced in NCAPGoverexpressing cells, while it was inhibited in NCAPG- silenced cells (Fig. 6d). Simultaneously, ectopic NCAPG increased, while silencing NCAPG decreased, the phosphorylation of STAT3, as well as the expression of STAT3-signaling downstream factors, Cyclin D1 and BCL2, which are markers for cell proliferation and antiapoptotic capacity, respectively (Fig. 6b). Furthermore, the nuclear localization of STAT3 was found to be promoted by NCAPG overexpression and suppressed by NCAPG silencing (Figs. 6e and S6). The mRNA expression of STAT3 downstream genes was also upregulated by NCAPG (Fig. 6f). Collectively, these results suggest that NCAPG exerts trastuzumab resistance through activation of the SRC/STAT3-signaling pathway. 


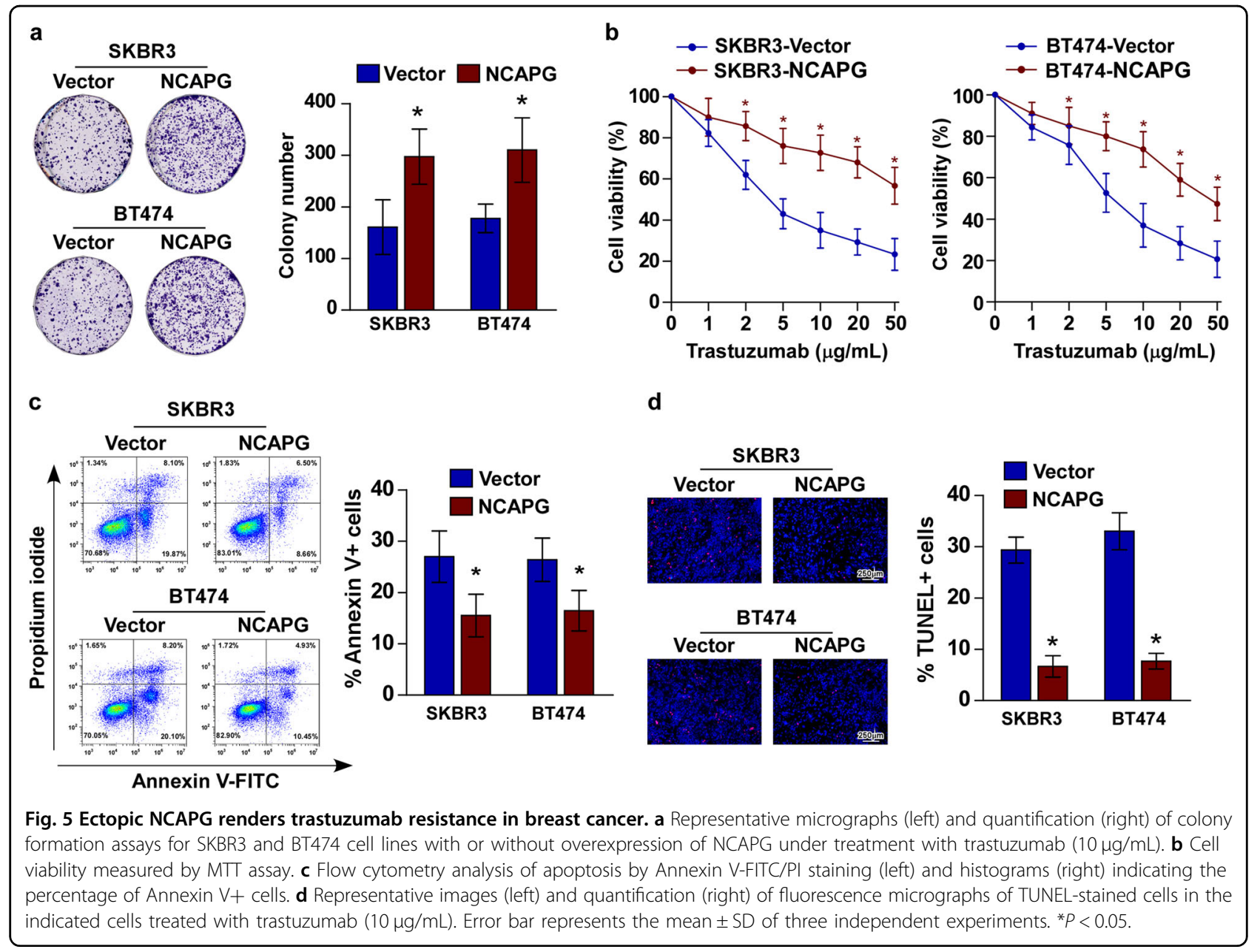

NCAPG expression correlates clinically with the activation of SRC/STAT3 signaling in BC

Analysis of IHC staining revealed that enhanced expression of NCAPG significantly correlated with the phosphorylation of SRC (Tyr416) and elevated nuclear localization of STAT3 in BC samples (Fig. 7a, b). Moreover, western blotting and EMSA assay proved that NCAPG expression promoted the phosphorylation of SRC (Tyr416) $(r=0.831, P=0.002)$ and STAT3 transcriptional activity $(r=0.804, P=0.003)$ (Fig. 7c, d). Overall, these results suggest that NCAPG induces trastuzumab resistance in HER2 $+\mathrm{BC}$ by activation of SRC/ STAT3 signaling.

\section{Discussion}

Trastuzumab was the first anti-HER2 agent to be approved by the US Food and Drug Administration (FDA) and remains the backbone for treatment of HER2 $+B C$ with the high selectivity ${ }^{23}$. Although trastuzumab is well tolerated, there is increasing clinical data showing trastuzumab resistance in HER2 $+\mathrm{BC}$ therapy ${ }^{8,9}$. Although trastuzumab combined with chemotherapy drugs can improve the therapeutic efficacy of patients, the mild and moderate adverse reactions after combined chemotherapy significantly increased ${ }^{24,25}$. Moreover, with the occurrence of trastuzumab resistance, the response duration of combined treatment is only $5-9$ months ${ }^{26}$. New drugs are developed to overcome trastuzumab resistance in HER2+ $\mathrm{BC}$, such as lapatinib and pertuzumab, etc. However, due to the single target, limited benefit or the dependency of trastuzumab, most of the alternative drugs cannot completely replace trastuzumab in efficacy and safety ${ }^{27,28}$. Trastuzumab is still the best first-line drug for HER2 + BC patients.

Trastuzumab blocks dimerization and activation of the HER2 receptor by binding the extracellular domain of HER2, inhibits shedding of the HER2 extracellular region to inactivate multiple intracellular signaling pathways, and mediates antibody-induced cytotoxicity to inhibit the proliferation of tumor cells ${ }^{29,30}$. Previous research found that effective interruption of the binding between trastuzumab and HER2 is one of the most common 


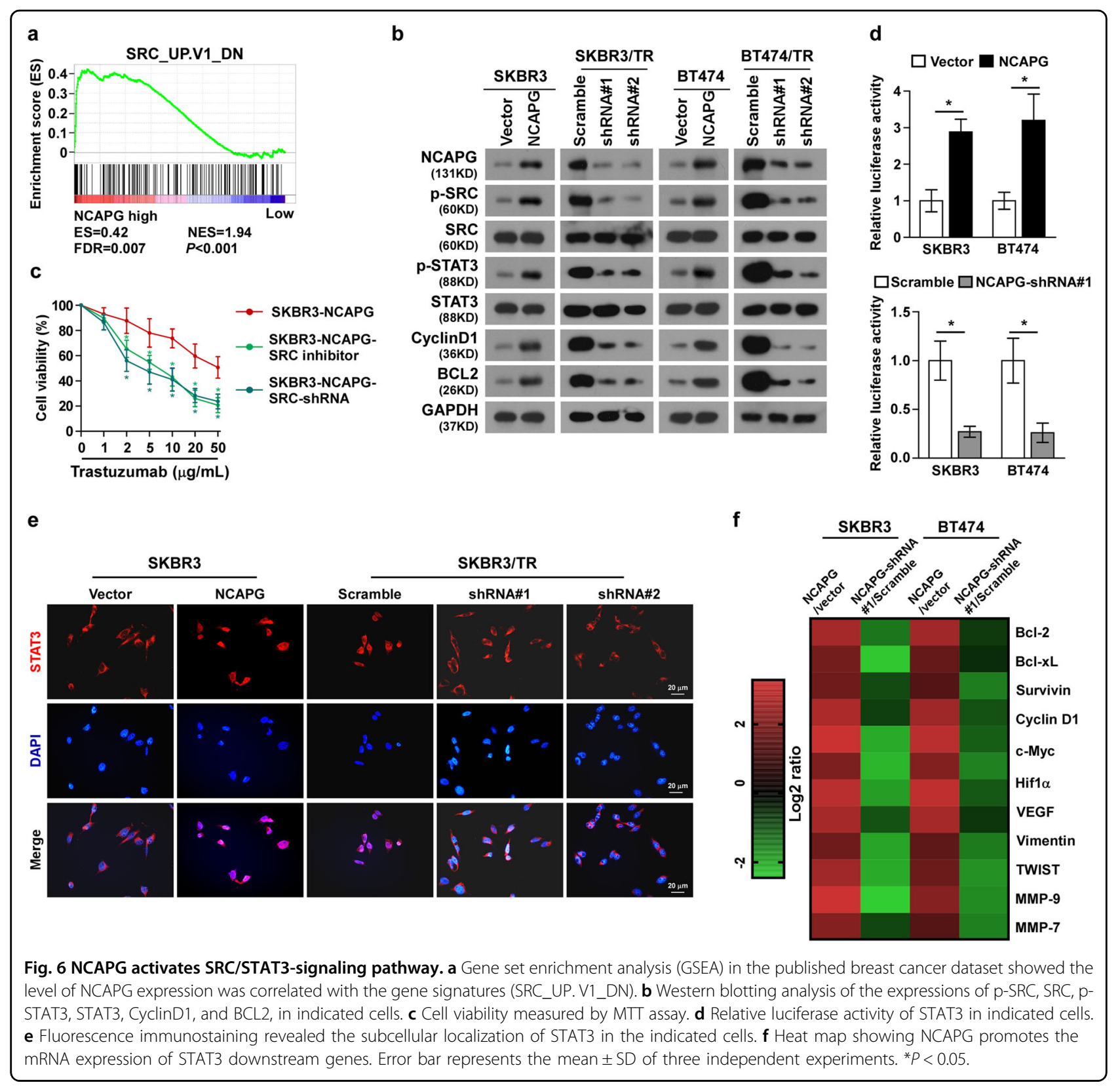

mechanisms of trastuzumab resistance ${ }^{31}$. The constitutive activation of p95HER2, the truncated form of the HER2 receptor, and high expression of membrane-related glycoprotein mucin 4 (MUC4) were both found to inhibit the binding of trastuzumab to HER2 ${ }^{32,33}$. However, even if trastuzumab binds effectively to the HER2 extracellular segment, drug resistance can still occur through intracellular activation of signal pathways, resulting in tumor progression $^{34}$. In line with this, our study found that the intracellular oncogene NCAPG is significantly overexpressed in HER2+ BC, particularly in patients' samples and $\mathrm{BC}$ cell lines which are trastuzumab-resistant. Upregulation of NCAPG indicates poor survival and relapse in
HER2 + trastuzumab-resistant BC, while knockdown of NCAPG enables re-sensitization of resistant HER2+ BC cells to trastuzumab.

NCAPG has been previously reported as a tumorpromoting gene and facilitates tumor progression. It was previously found that NCAPG promotes proliferation, metastasis, and recurrence in $\mathrm{HCC}^{12}$. RNA sequencing revealed that overexpression of NCAPG can induce cell proliferation and inhibit apoptosis via activation of the PI3K-AKT signaling pathway in $\mathrm{HCC}^{17}$. Upregulated NCAPG also correlated positively with the cell proliferation and poor survival in $\mathrm{BC}$ as well as the poor diseasefree survival and advanced clinical stage in castration- 


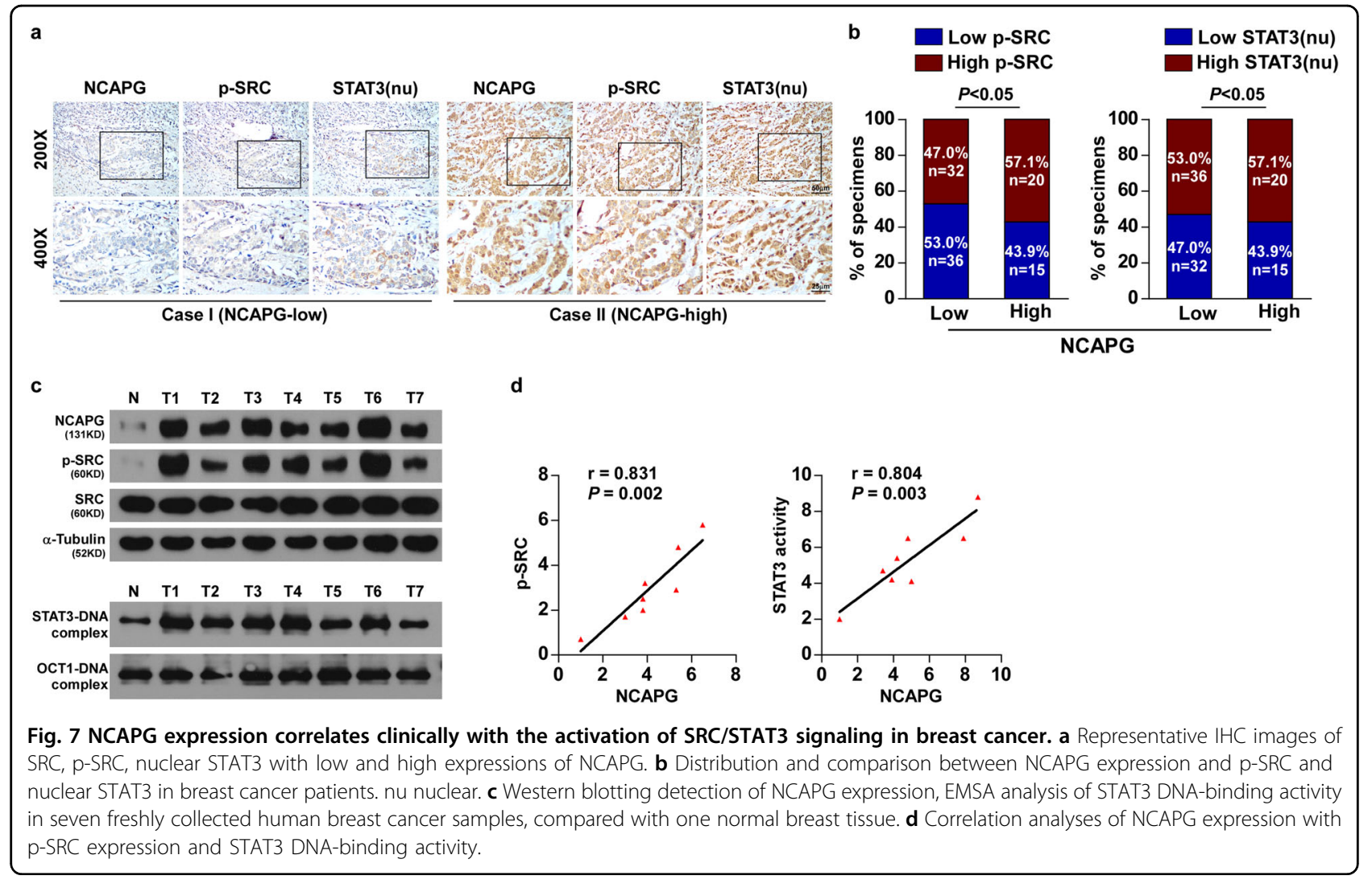

resistant prostate cancer ${ }^{14,18,19}$. Silencing NCAPG was shown to enhance the effect of temozolomide on inhibition of cell proliferation in pediatric high grade gliomas ${ }^{13}$. However, to our knowledge, no in-depth study investigating NCAPG in BC drug resistance has been carried out. Strikingly, NCAPH, another subunit of condensin 1, which has also been shown to be a tumor-promoting factor, is expressed in hormone-sensitive prostate cancer and involved in resistance to castration ${ }^{35}$. Besides, overexpression of NCAPH has been found to be associated with carboplatin resistance in ovarian cancer ${ }^{36}$. Recently, Shimomura et al. revealed that NCAPH confers platinum resistance to oral squamous cell carcinoma cells, and NCAPG was found to interact with $\mathrm{NCAPH}^{37}$. These studies indicated a potential role of NCAPG in conferring drug resistance. Consistently, our study demonstrated that expression of NCAPG is increased in trastuzumabresistant $\mathrm{BC}$, and NCAPG overexpression promotes the proliferative and anti-apoptotic cell features, while silencing NCAPG re-sensitized the response to trastuzumab in trastuzumab-resistant HER2+ BC.

Previous studies demonstrated that SRC is elevated and constitutively activated in multiple cancers containing breast, colon, lung, and pancreas ${ }^{38}$. Extracellular signals such as PDGFR, HER family members (HER1; HER2 and HER3), and IGF1R can phosphorylate SRC on Tyr416, which is followed by activation of MAPK, PI3K, and STAT3-signaling pathways and results in cell differentiation and proliferation ${ }^{39,40}$. The activation of the SRC, PI3K/AKT, and STAT3 pathway can facilitate the proliferation and growth of tumor cells by upregulating the expression of Myc, CyclinD1, and CDC2; inhibit apoptosis by upregulating BCL-xL, Survivin, and $\mathrm{Mcl}$; promote metastasis by upregulating FAK, p130Cs, MMP2, and MMP9; and accelerate tumor angiogenesis by upregulating VEGF and HIF1 $\alpha^{41-43}$. These biological processes are all important mechanisms of trastuzumab resistance in $\mathrm{BC}^{44}$. It has been shown that SRC activation confers resistance to the HER2/EGFR tyrosine kinase inhibitor lapatinib ${ }^{45}$, while knockdown of SRC was found to sensitize response to PI3K $\alpha$ inhibitor alpelisib in ER+ BC cells ${ }^{46}$. Additionally, the constitutive activation of STAT3 was reported to be accompanied by overexpression and activation of SRC in BC, resulting in chemotherapeutic resistance $^{47}$. SRC inhibitors were shown to reduce STAT3 activity and sensitize the response to Taxol in HER2overexpressing $\mathrm{BC}^{48}$. All these studies suggest SRC/ STAT3 signaling could be a potential target of trastuzumab-resistant BC. Herein, we found that NCAPG confers trastuzumab resistance by activating the SRC/ STAT3-signaling pathway, which demonstrates a novel mechanism of trastuzumab resistance and provide the 
potential target for therapy. The further mechanism of NCAPG activating the SRC/STAT3 is necessary and has been under our investigation. It has been reported that HER2-induced secretion of IL-6 can act in an autocrine fashion in human tumor cells causes STAT3-mediated gene expression and signaling activation and facilitates oncogenic growth $^{49}$. Lu et al. found a binding site of STAT3 to HER2 promoter and observed a stimulatory effect both on HER2 mRNA and protein expressions in MCF-7 cells stably expressing STAT3, suggesting that STAT3 upregulated HER2 expression ${ }^{50}$. Collectively, HER2 might take part in the regulation of NCAPG to SRC/STAT3 and a potential positive feedback loop regulation mechanism may exist in this regulatory network.

In conclusion, our study revealed that NCAPG promotes trastuzumab resistance, at least in part via SRC/ STAT3 pathway activation. Our results demonstrate a critical role for NCAPG in conferring trastuzumab resistance and suggest that NCAPG may be a potential therapeutic target against trastuzumab resistance in HER2 + BC.

\section{Materials and methods}

\section{Cell lines and cell culture}

The human HER2+ BC cell lines, SKBR3 and BT474, were purchased from ATCC (Manassas, VA, USA). SKBR3 cells were cultured in McCoy'5A (GIBCO, Grand Island, NY) containing $10 \%$ fetal bovine serum (FBS) (HyClone, Logan, UT), and BT474 cell lines were cultured in DMEM (GIBCO) medium supplemented with $10 \%$ FBS in a humidified environment with $5 \% \mathrm{CO}_{2}$ at $37^{\circ} \mathrm{C}$. All cell passaging or performing experiments were performed while cells reached $50-80 \%$ density. $1 \times 10^{6}$ cells were passed into $10 \mathrm{~cm}$ culture dishes and incubated for $24 \mathrm{~h}$ ahead of trastuzumab exposure. The cells were continuously treated with trastuzumab in concentration of $10,50,100 \mu \mathrm{g} / \mathrm{mL}$. The process was conducted for $\sim 6$ months to induce trastuzumab-resistant SKBR3/TR and BT474/TR cells. Trastuzumab is a gift from the Sun Yat-sen University Cancer Center. The SRC inhibitor, PP2 $(10 \mu \mathrm{M})$, was purchased from Selleck Chemicals (Houston, TX).

\section{BC biopsies}

This study collected $12 \mathrm{BC}$ biopsies from patients who had been treated with trastuzumab at the Sun Yat-sen University Cancer Center from 2002 to 2007. The biopsies were acquired prior to the initiation of trastuzumab. Tumor response to therapy defined as either complete response $(\mathrm{CR})$, partial response (PR), stable disease (SD), or progressive disease (PD), which was notarized by computed tomography and evaluated in line with the Response Evaluation Criteria in Solid tumors (RECIST) guidelines. The six cases with CR or PR were defined as trastuzumab sensitivity, whereas the other six cases with SD or PD were considered trastuzumab resistance. This study was licensed by the Institutional Research Ethics Committee, and the informed consents were obtained before using the specimens.

\section{IHC assay}

IHC staining was performed on the 103 paraffinembedded $\mathrm{BC}$ tissue sections with trastuzumab treatment, using anti-NCAPG, anti-p-SRC (Tyr416), and antip-STAT3 (Tyr705) antibodies. Two independent pathologists, blinded to the histopathological features and patient data, separately reviewed and scored the degree of immunostaining of the sections. IHC-staining intensity was scored: strong (brown): +3 ; moderate (yellow brown): +2 ; weak (light yellow): +1 ; and negative (no staining): 0 . The proportion of tumor cells was evaluated as follows: 0 (no staining tumor cells), 1 ( $<10 \%$ staining tumor cells), 2 (10-50\% staining tumor cells), and 3 (>50\% staining tumor cells). The staining index was scored as staining intensity by the proportion of tumor cells. The cut off value was the median value 3 . Staining index score $0,1,2$, 3 were defined as low expression, whereas those that scored 4, 6, 9 were considered as high expression. Specimens appearing $>10 \%$ nuclear expression were considered nuclear positive.

\section{Constructs, transfection, and retroviral infection}

The plasmids pLV-EF1A-hNCAPG and pLV[shRNA]U6-hNCAPG (shRNA\#1 and shRNA\#2) were purchased from VectorBuilder (VectorBuilder Inc., Guangdong, China). Lipofectamine 3000 reagent (Invitrogen, Carlsbad, CA) was used for transfection according to the manufacturer's instructions. Stable cell lines expressing NCAPG or NCAPG-shRNAs were selected over 10 days using $0.5 \mu \mathrm{g} / \mathrm{mL}$ puromycin. Cell lysates were prepared to RNA/protein extraction for further experiments.

\section{RNA extraction, reverse transcription, and qPCR}

Total RNA was isolated using Trizol reagent (Invitrogen). RNA was quantified using a NanoDrop ND-1000. Reverse transcription-PCR was implemented using a SuperScript One-Step RT-PCR with Platinum Taq (Invitrogen) and $1 \mu \mathrm{g}$ RNA in a Bio-Rad MyCyler (Bio-Rad, Hercules, CA). Quantitative real-time PCR (qPCR) was conducted using the FastStart Universal SYBR Green Master (Roche, Toronto, ON, Canada) with a Bio-Rad CFX96 Real Time System C1000 Cycler (Bio-Rad). The levels of gene expression were normalized to the housekeeping gene, GAPDH, using CFX Manager V2 software.

\section{Western blotting analysis}

RIPA lysis buffer supplemented with a Protease/Phosphatase Inhibitor Cocktail (Cat\# 5872, Cell Signaling 
Technology, Danvers, MA) was used to extract from both the tissue and cell samples. The protein was quantified using a bicinchoninic acid (BCA) assay (Pierce, Rockford, IL) according to the manufacturer's instructions. The same amount $(30 \mu \mathrm{g})$ protein of each sample was separated using SDS-PAGE and transferred to polyvinylidene difluoride membranes (Roche). The membranes were blocked with $5 \%$ nonfat milk in TBST (0.1\% Tween-20) for $1 \mathrm{~h}$ at room temperature, and subsequently incubated with the appropriate primary antibodies overnight at $4{ }^{\circ} \mathrm{C}$. After washing three times in TBST, the membranes were incubated with horseradish peroxidase (HRP)-conjugated secondary antibodies (1:5000, Cat\# ab7090, Cat\# ab97040, Abcam, Cambridge, MA) for $1 \mathrm{~h}$ at room temperature. The following antibodies were used: anti-NCAPG rabbit polyclonal antibody (1:1000, Cat\# 24563-1-AP, PROTEINTECH, Hubei, China), and anti-p84 (Cat\# ab102684, Abcam), anti-STAT3 (Cat\# ab119352, Abcam), antipSTAT3 (Tyr705, Cat\# ab76315, Abcam), and anti-SRC (Cat\# 2109), anti-p-SRC (Tyr416, Cat\#2101), anti-Cyclin D1 (Cat\# 55506), anti-BCL2 (Cat\# 15071), anti-GAPDH (Cat\# 5174), and anti- $\alpha$-Tubulin (Cat\# 2125) (1:1000, Cell Signaling Technology).

\section{Colony formation assay}

Cells were seeded into a six-well culture plates at a density of $1.0 \times 10^{3}$ cells and grown in adaptive medium in a humidified environment with $5 \% \mathrm{CO}_{2}$ at $37^{\circ} \mathrm{C}$ for $18 \mathrm{~h}$, then replaced the medium by a fresh medium containing $10 \mu \mathrm{g} / \mathrm{mL}$ trastuzumab for incubating for 14 days. After the culture period, discarded the culture and fixed the cells by $4 \%$ polyoxymethylene for $30 \mathrm{~min}$ then stained cells with crystal violet, and counted under an inverted microscope.

\section{Annexin V-FITC/propidium iodide (PI)-stained assay}

Cells were seeded into a six-well plate at a density of $60 \%$ for analyzing the cellular apoptotic rate using flow cytometry according to the standard manufacturer protocol (MACS Miltenyi Biotec, Germany). The cells were trypsinized, washed with ice-cold PBS, and centrifuged at $1000 \times g$ for $5 \mathrm{~min}$, followed by re-suspension in binding buffer at a density of $1.0 \times 10^{6}$ cells $/ \mathrm{mL}$. Subsequently, the cells were incubated with Annexin V-isothiocyanate fluorescein and PI (BD, CA) for $25 \mathrm{~min}$ at $4{ }^{\circ} \mathrm{C}$ in dark. After that, the stained cells were analyzed using Cytomics FC500 (Beckman Coulter, Miami, FL) at an excitation wavelength of $488 \mathrm{~nm}$. Apoptotic cells were the Annexin V-positive cells.

\section{Terminal transferase dUTP nick end labeling (TUNEL) assays}

The indicated cells and tissues were fixed with paraformaldehyde. The TUNEL Assay Kit was used to assess the cell apoptosis according to the manufacturer's instruction (KeyGEN, Guangdong, China). In brief, cells or tissues were fixed in $4 \%$ paraformaldehyde for $30 \mathrm{~min}$ at room temperature, washed three times with PBS and permeabilized with $0.1 \%$ Triton-X 100 for $5 \mathrm{~min}$ at room temperature. Then the samples were stained with Streptavidin-TRITC under the action of TdT enzyme for $30 \mathrm{~min}$ at $37^{\circ} \mathrm{C}$, washed three times with PBS, and counterstained cell nuclei with DAPI. The images were obtained with fluorescence microscope (Leica, Buffalo Grove, IL).

\section{Tumor xenografts}

All animal experiments were approved by the Institutional Animal Care and Use Committee of Sun Yat-sen University. Animals were randomly divided into groups and the experiments were performed independently and blindly. Briefly, $2 \times 10^{6}$ cells (SKBR3/TR-Scramble, SKBR3/TR-shRNA\#1, SKBR3/TR-shRNA\#2) were subcutaneously injected into the mammary fat pad of 4-weekold female BALB/c nude mice (19-22 g). When the average size of tumor reached $100 \mathrm{~mm}^{3}$, the mice were injected with trastuzumab $(10 \mathrm{mg} / \mathrm{kg}$, once a week) intraperitoneally for 4 weeks. The mice weight was measured every week. The tumor volume was calculated using the equation: $\left(L \times W^{2}\right) / 2$. The mice were sacrificed after 4week treatment, and the tumors were harvested, weighed, and photographed. Serial $6.0 \mu \mathrm{m}$ sections were sliced and underwent TUNEL (KeyGEN) staining to analyze the apoptosis rate.

\section{Luciferase reporter assays}

Cells were seeded in triplicate into 24-well plates at a density of $5 \times 10^{4} .500 \mathrm{ng}$ STAT3 luciferase reporters plus $5 \mathrm{ng}$ pRL-TK Renilla plasmid (Promega, Madison, WI) were transfected into indicated cells using Lipofectamine 3000 reagent (Invitrogen) according to the manufacturer's instruction. The luciferase and Renilla signals were measured at $36 \mathrm{~h}$ after transfection using a Dual Luciferase Reporter Assay Kit (Promega). The relative luciferase activity was calculated by normalizing to the Renilla signal.

\section{Immunofluorescence (IF) staining}

Cells $\left(5 \times 10^{4}\right)$ were cultured on slides for $24 \mathrm{~h}$, washed with PBS for three times and treated with $1 \%$ Triton X100. The cells were stained with anti-STAT3 (Cell Signaling Technology), for $2 \mathrm{~h}$ at $4{ }^{\circ} \mathrm{C}$, washed with PBS, and incubated with a TRITC-conjugated secondary antibody (1:100, Cell Signaling Technology) at $37^{\circ} \mathrm{C}$ for $1 \mathrm{~h}$. The cell nuclei were visualized by counterstaining with DAPI (25 ng/mL, Cat\#, D9542, Sigma, St. Louis, MO). The images were obtained with a confocal laser-scanning microscope (Carl Zeiss, Oberkochen, Germany). 


\section{Electrophoretic mobility shift assay (EMSA)}

EMSA was performed according to the manufacturer's protocol using a LightShift Chemiluminescent EMSA kit (Pierce Biotechnology, Rockford, IL). DNA probes containing specific binding sites were used: STAT3: sense, 5'-GATCCTTCTGGGAATTCCTAGATC-3', antisense, 5'-GATCTAGGAATTCCCAGAAGGATC-3'; OCT-1 (used as the negative control): sense, $5^{\prime}$-TGTCGAATGC AAATCACTAGAA- $3^{\prime}$, antisense, $5^{\prime}$-TTCTAGTGATTT G CATTCGACA- $3^{\prime}$.

\section{Statistical analysis}

Data were analyzed using the SPSS version 19.0 statistical software package. The statistical tests used to analyze the data included a log-rank test, $\chi^{2}$ test, Spearman-rank correlation test, and Student's $t$ test (two-sided). The data are presented as the mean $\pm \mathrm{SD}$. A threshold of $P<0.05$ indicated statistical significance.

\begin{abstract}
Acknowledgements
This work was supported by the Natural Science Foundation of China (Nos. 81972619 and 81672874), the Basic and Applied Research Projects of Guangzhou Science and Technology Bureau (202002030067), the Distinguished Young Scholar of Guangdong Province (No. 2015A030306033), the Young Scholar of Science and Technology of Guangdong Province (2016TQ03R801), the Innovative Academic Team of Guangzhou Education System (1201610014), the Science and Technology Program of Guangzhou (201604020001 and 201803010098), the Natural Science Foundation research team of Guangdong Province (2018B030312001), the Research Team of Department of Education of Guangdong Province (2017KCXTD027), the Medical Science and Technology Research Foundation of Guangdong Province (A2020403), the Guangzhou key medical discipline construction project fun, Guangzhou traditional Chinese medicine and traditional Chinese and western medicine science and technology project (20182A011025).
\end{abstract}

\section{Author details \\ ${ }^{1}$ Affiliated Cancer Hospital \& Institute of Guangzhou Medical University, 510095 Guangzhou, China. ${ }^{2}$ Guangzhou Municipal and Guangdong Provincial Key Laboratory of Protein Modification and Degradation, School of Basic Medical Science, Guangzhou Medical University, 511436 Guangzhou, China. ${ }^{3}$ Department of Biochemistry, Zhongshan School of Medicine, Sun Yat-sen University, 510080 Guangzhou, China. ${ }^{4}$ Department of Ultrasonography, Guangzhou Women and Children's Medical Center, Guangzhou Medical University, 510623 Guangzhou, China. 5 Department of Urologic Oncosurgery, Affiliated Cancer Hospital \& Institute of Guangzhou Medical University, 510095 Guangzhou, China. ${ }^{6}$ Department of Pathogen Biology and Immunology, School of Basic Courses, Guangdong Pharmaceutical University, 510006 Guangzhou, China. ${ }^{7}$ Key Laboratory of Molecular Target \& Clinical Pharmacology, School of Pharmaceutical Sciences \& the Fifth Affiliated Hospital, Guangzhou Medical University, 511436 Guangzhou, China. ${ }^{8}$ State Key Laboratory of Oncology in South China, Collaborative Innovation Center for Cancer Medicine, Sun Yat-sen University Cancer Center, 510060 Guangzhou, China}

\section{Conflict of interest}

The authors declare that they have no conflict of interest.

\section{Publisher's note}

Springer Nature remains neutral with regard to jurisdictional claims in published maps and institutional affiliations.

Supplementary Information accompanies this paper at (https://doi.org/ 10.1038/s41419-020-02753-x).
Received: 26 March 2020 Revised: 30 June 2020 Accepted: 3 July 2020

Published online: 18 July 2020

\section{References}

1. Bray, F. et al. Global cancer statistics 2018: GLOBOCAN estimates of incidence and mortality worldwide for 36 cancers in 185 countries. CA Cancer J. Clin. 68, 394-424 (2018).

2. Perou, C. M. et al. Molecular portraits of human breast tumours. Nature 406, 747-752 (2000).

3. Curtis, $C$. et al. The genomic and transcriptomic architecture of 2,000 breast tumours reveals novel subgroups. Nature 486, 346-352 (2012).

4. Ross, J. S. et al. The HER-2 receptor and breast cancer: ten years of targeted anti-HER-2 therapy and personalized medicine. Oncologist 14, 320-368 (2009).

5. Slamon, D. J. et al. Human breast cancer: correlation of relapse and survival with amplification of the HER-2/neu oncogene. Science 235, 177-182 (1987).

6. Cameron, D. et al. 11 years' follow-up of trastuzumab after adjuvant chemotherapy in HER2-positive early breast cancer: final analysis of the HERceptin Adjuvant (HERA) trial. Lancet 389, 1195-1205 (2017).

7. Rimawi, M. F., Schiff, R. \& Osborne, C. K. Targeting HER2 for the treatment of breast cancer. Annu. Rev. Med. 66, 111-128 (2015).

8. Valabrega, G., Montemurro, F. \& Aglietta, M. Trastuzumab: mechanism of action, resistance and future perspectives in HER2-overexpressing breast cancer. Ann. Oncol. 18, 977-984 (2007).

9. Nahta, R. \& Esteva, F. J. HER2 therapy: molecular mechanisms of trastuzumab resistance. Breast Cancer Res. 8, 215 (2006).

10. Lammens, A., Schele, A. \& Hopfner, K. P. Structural biochemistry of ATP-driven dimerization and DNA-stimulated activation of SMC ATPases. Curr. Biol. 14, 1778-1782 (2004)

11. Palou, R. et al. Condensin ATPase motifs contribute differentially to the maintenance of chromosome morphology and genome stability. PLoS Biol. 16, e2003980 (2018).

12. Liu, W. et al. Overexpression of nonSMC condensin I complex subunit G serves as a promising prognostic marker and therapeutic target for hepatocellular carcinoma. Int. J. Mol. Med. 40, 731-738 (2017).

13. Liang, M. L. et al. Downregulation of miR-137 and miR-6500-3p promotes cell proliferation in pediatric high-grade gliomas. Oncotarget 7, 19723-19737 (2016).

14. Arai, T. et al. Regulation of NCAPG by miR-99a-3p (passenger strand) inhibits cancer cell aggressiveness and is involved in CRPC. Cancer Med. 7, 1988-2002 (2018).

15. Song, B., Du, J., Song, D. F., Ren, J. C. \& Feng, Y. Dysregulation of NCAPG, KNL1, miR-148a-3p, miR-193b-3p, and miR-1179 may contribute to the progression of gastric cancer. Biol. Res. 51, 44 (2018).

16. Zhang, Q., Su, R., Shan, C., Gao, C. \& Wu, P. Non-SMC Condensin I complex, subunit G (NCAPG) is a novel mitotic gene required for hepatocellular cancer cell proliferation and migration. Oncol. Res. 26, 269-276 (2018).

17. Gong, C. et al. NCAPG promotes the proliferation of hepatocellular carcinoma through PI3KJAKT signaling. Onco Targets Ther. 12, 8537-8552 (2019).

18. Chen, J., Qian, X., He, Y., Han, X. \& Pan, Y. Novel key genes in triple-negative breast cancer identified by weighted gene co-expression network analysis. J. Cell. Biochem. 120, 16900-16912 (2019).

19. Hitti, E. et al. Systematic analysis of AU-rich element expression in cancer reveals common functional clusters regulated by key RNA-binding proteins. Cancer Res. 76, 4068-4080 (2016).

20. Hamberg, P. et al. Randomized phase II study comparing efficacy and safety of combination-therapy trastuzumab and docetaxel vs. sequential therapy of trastuzumab followed by docetaxel alone at progression as first-line chemotherapy in patients with HER2+ metastatic breast cancer: HERTAX trial. Clin. Breast Cancer 11, 103-113 (2011).

21. Nagy, A., Lánczky, A., Menyhárt, O.\& Gyorffy, B. Validation of miRNA prognostic power in hepatocellular carcinoma using expression data of independent datasets. Sci. Rep. 8, 9227 (2018).

22. Tan, Q. et al. Src/STAT3-dependent heme oxygenase-1 induction mediates chemoresistance of breast cancer cells to doxorubicin by promoting autophagy. Cancer Sci. 106, 1023-1032 (2015).

23. Moasser, M. M. \& Krop, I. E. The evolving landscape of HER2 targeting in breast cancer. JAMA Oncol. 1, 1154-1161 (2015).

24. Slamon, D. J. et al. Use of chemotherapy plus a monoclonal antibody against HER2 for metastatic breast cancer that overexpresses HER2. N. Engl. J. Med. 344, 783-792 (2001). 
25. Burstein, H. J. et al. Trastuzumab and vinorelbine as first-line therapy for HER2overexpressing metastatic breast cancer: multicenter phase II trial with clinical outcomes, analysis of serum tumor markers as predictive factors, and cardiac surveillance algorithm. J. Clin. Oncol. 21, 2889-2895 (2003).

26. Cobleigh, M. A. et al. Multinational study of the efficacy and safety of humanized anti-HER2 monoclonal antibody in women who have HER2overexpressing metastatic breast cancer that has progressed after chemotherapy for metastatic disease. J. Clin. Oncol. 17, 2639-2648 (1999).

27. Petrelli, F. et al. The efficacy of lapatinib and capecitabine in HER-2 positive breast cancer with brain metastases: a systematic review and pooled analysis. Eur. J. Cancer (Oxf., Engl.: 1990) 84, 141-148 (2017).

28. Gianni, L. et al. Neoadjuvant treatment with trastuzumab and pertuzumab plus palbociclib and fulvestrant in HER2-positive, ER-positive breast cancer (NA-PHER2): an exploratory, open-label, phase 2 study. Lancet Oncol. 19, 249-256 (2018).

29. Hudis, C. A. Trastuzumab-mechanism of action and use in clinical practice. $N$. Engl. J. Med. 357, 39-51 (2007).

30. Molina, M. A. et al. Trastuzumab (herceptin), a humanized anti-Her2 receptor monoclonal antibody, inhibits basal and activated Her2 ectodomain cleavage in breast cancer cells. Cancer Res. 61, 4744-4749 (2001).

31. Pohlmann, P. R., Mayer, I. A. \& Mernaugh, R. Resistance to Trastuzumab in breast cancer. Clin. Cancer Res. 15, 7479-7491 (2009).

32. Scaltriti, M. et al. Expression of p95HER2, a truncated form of the HER2 receptor, and response to anti-HER2 therapies in breast cancer. J. Natl. Cancer Inst. 99, 628-638 (2007).

33. Carraway, K. L., Theodoropoulos, G., Kozloski, G. A. \& Carothers Carraway, C. A. Muc4/MUC4 functions and regulation in cancer. Future Oncol. 5, 1631-1640 (2009).

34. Grell, P., Svoboda, M., Simickova, M., Fabian, P. \& Vyzula, R. [Trastuzumab in the breast cancer treatment: efficacy and resistance mechanisms]. Klin. Onkol. 22, 45-51 (2009).

35. Arai, T. et al. Micro-ribonucleic acid expression signature of metastatic castration-resistant prostate cancer: regulation of NCAPH by antitumor miR199a/b-3p. Int. J. Urol. 26, 506-520 (2019).

36. Zhan, S. J., Liu, B. \& Linghu, H. Identifying genes as potential prognostic indicators in patients with serous ovarian cancer resistant to carboplatin using integrated bioinformatics analysis. Oncol. Rep. 39, 2653-2663 (2018).
37. Shimomura, H. \& Sasahira, T. Non-SMC condensin I complex subunit $H$ (NCAPH) is associated with lymphangiogenesis and drug resistance in oral squamous cell carcinoma. J. Clin. Med. 9, 72 (2019).

38. Dehm, S. M. \& Bonham, K. SRC gene expression in human cancer: the role of transcriptional activation. Biochem. Cell Biol. = Biochim. Biol. Cell. 82, 263-274 (2004).

39. Olayioye, M. A., Badache, A., Daly, J. M. \& Hynes, N. E. An essential role for Src kinase in ErbB receptor signaling through the MAPK pathway. Exp. Cell Res. 267, 81-87 (2001)

40. Mitra, S. K. \& Schlaepfer, D. D. Integrin-regulated FAK-Src signaling in normal and cancer cells. Curr. Opin. Cell Biol. 18, 516-523 (2006).

41. Ebbesen, S. H. et al. Pten loss promotes MAPK pathway dependency in HER2/ neu breast carcinomas. Proc. Natl Acad. Sci. USA 113, 3030-3035 (2016).

42. Malek, M. et al. PTEN regulates PI(3,4)P2 signaling downstream of class I PI3K. Mol. Cell 68, 566-580.e510 (2017).

43. Alvarez, R. H., Kantarjian, H. M. \& Cortes, J. E. The role of Src in solid and hematologic malignancies: development of new-generation Src inhibitors. Cancer 107, 1918-1929 (2006).

44. Ocana, A., Cruz, J. J. \& Pandiella, A. Trastuzumab and antiestrogen therapy: focus on mechanisms of action and resistance. Am. J. Clin. Oncol. 29, 90-95 (2006).

45. Rexer, B. N. et al. Phosphoproteomic mass spectrometry profiling links Src family kinases to escape from HER2 tyrosine kinase inhibition. Oncogene 30, 4163-4174 (2011).

46. Le, X. et al. Systematic functional characterization of resistance to PI3K Inhibition in breast cancer. Cancer Discov. 6, 1134-1147 (2016).

47. Tzeng, Y. T. et al. Kinome-wide siRNA screening identifies Src-enhanced resistance of chemotherapeutic drugs in triple-negative breast cancer cells. Front. Pharmacol. 9, 1285 (2018).

48. Hawthorne, V. S. et al. ErbB2-mediated Src and signal transducer and activator of transcription 3 activation leads to transcriptional up-regulation of p21Cip1 and chemoresistance in breast cancer cells. Mol. Cancer Res. 7, 592-600 (2009).

49. Hartman, Z. C. et al. HER2 overexpression elicits a proinflammatory IL-6 autocrine signaling loop that is critical for tumorigenesis. Cancer Res. 71, 4380-4391 (2011).

50. Qian, L. et al. A novel cis-acting element in Her2 promoter regulated by Stat3 in mammary cancer cells. Biochem. Biophys. Res. Commun. 345, 660-668 (2006). 